Les chrysanthèmes de M. Homais. Les commémorations de Gustave Flaubert en Normandie et à Paris (1890-1923)

Mr Homais' Mums: Commemorations of Flaubert in Normandy and in Paris (1890-1923)

Marie-Clémence Régnier

\title{
OpenEdition
}

Journals

Édition électronique

URL : https://journals.openedition.org/recherchestravaux/2003

DOI : 10.4000/recherchestravaux.2003

ISSN : 1969-6434

Éditeur

UGA Éditions/Université Grenoble Alpes

Édition imprimée

ISBN : 978-2-37747-197-3

ISSN : 0151-1874

Référence électronique

Marie-Clémence Régnier, «Les chrysanthèmes de M. Homais. Les commémorations de Gustave Flaubert en Normandie et à Paris (1890-1923) », Recherches \& Travaux [En ligne], 96 | 2020, mis en ligne le 23 juin 2020, consulté le 29 juin 2021. URL : http://journals.openedition.org/ recherchestravaux/2003; DOI : https://doi.org/10.4000/recherchestravaux.2003

Ce document a été généré automatiquement le 29 juin 2021.

(c) Recherches \& Travaux 


\title{
Les chrysanthèmes de $M$. Homais. Les commémorations de Gustave Flaubert en Normandie et à Paris (1890-1923)
}

\author{
Mr Homais' Mums: Commemorations of Flaubert in Normandy and in Paris \\ (1890-1923)
}

Marie-Clémence Régnier

1 Dans un ouvrage récent consacré aux commémorations d'écrivains européens à l'époque moderne ${ }^{1}$, Pierre Boudrot s'est intéressé à la dimension polémique de la culture commémorative française. Étudiant les commémorations organisées en 1878 en l'honneur de Voltaire, l'historien remarque qu'il s'agit là d'un «usage de la commémoration particulièrement polémique », faisant montre d'une «culture de la contestation plutôt que de l'unité ${ }^{2}$. Si ce trait n'est pas propre à la France - l'ouvrage mentionné traite des débats entourant presque chaque date-anniversaire-, il n'en demeure pas moins que l'organisation de commémorations en France fait couramment l'objet de débats animés dont quelques exemples contemporains témoignent avec éclat (Céline, Maurras $\left.{ }^{3} . ..\right)$.

2 Le cas de Gustave Flaubert s'avère dans cette perspective des plus singuliers: la mémoire du romancier ne fait guère polémique pour des raisons politiques ou idéologiques (même si l'on connaît les réticences de Flaubert vis-à-vis de la République démocratique), contrairement aux auteurs mentionnés. Si les commémorations en son honneur suscitent de vifs débats, c'est que Flaubert apparaît comme le porte-voix des critiques et des opposants à ce type de manifestations. L'écrivain ne s'est pas seulement érigé contre l'iniquité et l'artificialité de ces grand-messes collectives, manifestations de la Bêtise bourgeoise, dans son œuvre comme dans sa correspondance ${ }^{4}$; il apparaît aussi pendant longtemps comme un auteur non "commémorable » aux autorités de la "petite patrie» normande dont il est pourtant l'un des grands noms avec Pierre Corneille et Guy de Maupassant. Au purgatoire de la reconnaissance de son 
vivant et après sa mort, Flaubert y est considéré comme le contempteur des vices de la bourgeoisie rouennaise. Enfin et surtout, la profession de foi de l'« ermite de Croisset ", qui prône l'impersonnalité de l'artiste devant s'effacer au profit de son œuvre, suffit à poser le paradoxe qu'il y a à vouloir commémorer Gustave Flaubert, sur ses terres normandes comme ailleurs. À l'heure même où la culture de la commémoration s'épanouit comme jamais en France sous la Troisième République, le romancier fait figure d'épouvantail aux cortèges de la mémoire littéraire.

3 En plaçant ce texte à l'enseigne des «chrysanthèmes de M. Homais", fleurs emblématiques des cortèges mortuaires, nous étudierons les commémorations qui lui ont été consacrées après sa mort, à Paris et en Normandie. Pour ce faire, nous mettrons l'accent sur l'ancrage géographique de ces festivités et sur le caractère polémique, voire aporétique de ces commémorations célébrant une gloire littéraire rebelle à ces hommages.

\section{Comices commémoratifs}

4 Arrimées à l'exemple de Pierre Corneille et de ses maisons, la reconnaissance de Gustave Flaubert et les commémorations qui y participent empruntent au cours des décennies 1890-1920 un chemin semé d'embûches après une première phase qui, pourtant, laissait présager une heureuse fortune. La situation de Gustave Flaubert en Normandie s'avère des plus complexes. Perçu par la postérité et la critique littéraire comme un éminent représentant du caractère farouche des Normands et comme un excellent peintre des mœurs de sa région natale, le romancier est également réputé pour les critiques qu'il a adressées aux Rouennais. Aussi Flaubert est-il une gloire locale difficile à s'approprier. De fait, bien que sa renommée littéraire prohibe l'indifférence, sa position vis-à-vis des élus de Rouen rend difficiles les hommages publics à son égard ${ }^{5}$. Ainsi, les célébrations organisées autour de Flaubert passent toutes au tamis de la critique dans la presse locale et nationale entre 1890 et 1923. La presse satirique, tout particulièrement, s'amuse de l'incongruité de festivités solennelles jugées artificielles pour un écrivain qui, plus que tout autre, affichait ouvertement son hostilité vis-à-vis de ses compatriotes et de comices qui, d'« agricoles", sont devenus commémoratifs. Quant au Pavillon de Croisset, seul vestige de la maison familiale des Flaubert en périphérie de Rouen, il est un sujet de crispation avec les statues à l'effigie du maître de Croisset. Du succès relatif des commémorations de 1890, suivies par quelques Parisiens et quelques journalistes, au point culminant atteint lors du centenaire de 1921, les commémorations entourant le romancier prennent une ampleur en réalité toute paradoxale.

5 Au long du premier tiers du siècle, les proches de Flaubert et la critique littéraire se complaisent à souligner son identité régionale, quitte à dériver parfois du côté du régionalisme. Dans ses mémoires, Caroline Commanville dresse le portrait de son oncle en Normand "typique ", "enveloppé de la mélancolie des peuples du Nord ${ }^{6}$ " mais aussi « robuste $^{7} »$ comme un Viking. Ces caractéristiques sont mises en balance avec les racines champenoises de l'écrivain. Joëlle Robert a souligné à juste titre le lien étroit qu'entretient le texte de la nièce de Flaubert avec l'étude que Taine consacre à Jean de La Fontaine, lui-même champenois et déterminé par un tempérament mélancolique similaire. 
6 L'image normande de Flaubert se nourrit ainsi à la source des innombrables témoignages de ses proches qui avaient essaimé dans leur correspondance, dans leurs mémoires et dans la presse à la fin du siècle. Maxime du Camp parlait lui aussi du "géant» Flaubert dans ses Souvenirs littéraires". Mais la tendance s'accentue dans l'entre-deux-guerres, en particulier grâce à Albert Thibaudet. Dans son étude magistrale sur Flaubert, le critique décrit ainsi le tempérament de l'écrivain :

Flaubert est Normand [sic], bourgeois bourgeoisant de ce pays où il a constamment vécu, dont il s'est imprégné de partout, tant par la curiosité artistique qui l'inclinait vers lui que par les colères qui le levaient contre lui. Il était Normand intégral par son physique?.

7 Et de préciser, en établissant un lien de filiation entre Corneille et Flaubert, tous deux «Parisiens » par contrainte : «Corneille et lui sont deux beaux types d'indépendance normande, deux beaux refus que fait le sang nordique de s'adapter à la communauté de la capitale ${ }^{10} »$. C'est là rattacher opportunément la légende normande des deux grands écrivains à la légende nationale, grâce à la mention de leurs séjours parisiens. Parallèlement, la «normandisation » des maisons de Flaubert, conjointement à celle de son œuvre, est un ressort fondamental de la construction et de la réception du personnage de Flaubert en Normand accompli.

8 Mémoire collective et réception de l'écrivain et de son œuvre dans la critique s'articulent pour former un canon régional récupérable au-delà de la Normandie. Toujours d'actualité, la tendance s'accentue depuis que Georges Dubosc a publié un article dans Le Journal de Rouen en novembre 1890. Preuves à l'appui, il prétend avoir découvert à Ry les lieux véritables que le romancier aurait transposés dans Madame Bovary ${ }^{11}$. Thibaudet, dans le chapitre qu'il consacre à Madame Bovary, s'amuse justement du développement d'un marché touristique dans la ville qui prétend au statut de haut lieu littéraire : on peut y visiter les maisons des habitants ayant inspiré les personnages ou acheter des cartes postales ${ }^{12} \ldots$

\section{Le rôle central des proches et des admirateurs de l'écrivain}

Grâce à l'influence insistante des proches de l'écrivain, rassemblés en "Comité Flaubert » (Maupassant, Zola, Goncourt, son président, Daudet ou encore Tourguénieff), le romancier est aussi honoré dans sa ville natale au cours des décennies 1890 et 1900. Tout commence lorsque Charles Lapierre lance un appel public et une souscription dans la presse, le 13 mai 1880 , pour édifier un monument ${ }^{13}$. Le 14 mai, le maire, Alexandre Barrabé, relaie vainement l'idée lors du conseil municipal: il propose d'aménager une fontaine qui fasse pendant à celle de Louis Bouilhet ${ }^{14}$.

10 Le bas-relief de Henri Chapu est alors le premier monument édifié à la mémoire de Flaubert à Rouen ${ }^{15}$. Le monument annonce le début d'une prise en charge progressive de la mémoire de Flaubert en Normandie. Installé non pas à la bibliothèque mais au musée des beaux-arts, le bas-relief est offert par le comité de souscription à la ville. Il constitue le décor de la cérémonie solennelle qui a lieu le 23 novembre 1890. Edmond de Goncourt en a rapporté le déroulement dans un récit caustique : il y dénonce des discours qui accumulent «tous les clichés, tous les lieux communs, toutes les expressions éculées, toutes les homaiseries imaginables : un discours qui le fera battre par Flaubert le jour de la Résurrection ${ }^{16} »$. Cependant, la commémoration innove car 
elle donne à voir des manuscrits de Flaubert au musée des beaux-arts. En ce sens, celle de 1890 ouvre la voie au musée de Croisset.

11 Les autorités de la ville s'attachent à fédérer les habitants autour de l'écrivain, dans les discours au moins ${ }^{17}$. Pourtant la "population [serait] tout à fait indifférente à la cérémonie ${ }^{18}$ » selon certains témoins. Cela n'empêche pas la municipalité d'accepter à nouveau en 1907 les grâces d'un comité parisien présidé par le sous-secrétaire d'État aux beaux-arts Dujardin-Baumette, assisté par le rédacteur du Siècle, Maurice Guillemot ${ }^{19}$. Dans le contexte stimulant des commémorations entourant le tricentenaire de la naissance de Corneille en 1906 et du rachat de sa maison natale, les amis de Flaubert se mobilisent autour de leur champion et de deux monuments distincts: d'une part, une statue en pied du romancier par le sculpteur russe Léopold Bernstamm, installée rue Thiers (actuelle rue Jean Lecanuet ${ }^{20}$ ), d'autre part, l'organisation d'un pèlerinage au Pavillon de Croisset. Enfin, le rachat du Pavillon est décidé.

12 La propriété est remise en vente par un notaire rouennais, Maître Paul Toutain, connu dans le milieu littéraire sous le pseudonyme de Jean Revel. Pour favoriser la préservation du patrimoine de Flaubert, Toutain-Revel divise les titres de propriétés en deux lots, l'un concernant l'usine et ses dépendances, l'autre le pavillon du jardin et ce qui reste du parc. Le 20 novembre 1904, Georges Dubosc lance une campagne de presse dans le Journal de Rouen, pour racheter ce second lot et protéger de la démolition le dernier vestige du Croisset des Flaubert. Les sociétés érudites sont bientôt rejointes par les amoureux de la Normandie, des proches des Flaubert et des admirateurs, auxquels s'associent des écrivains renommés (José Maria de Heredia, Jules Claretie...), des savants (Albert Sorel), puis les représentants de la ville de Rouen. Pour que le pavillon ne soit pas une coquille vide, décision est prise d'y installer un musée. L'argumentaire de Toutain-Revel martèle l'adjectif qualificatif "grand» qui, désignant au départ l'écrivain et la nation, s'étend à la " petite patrie ${ }^{21}$ ». Ensuite, l'orateur réunit petite et grande patries autour de la figure fédératrice de Corneille à laquelle il rattache Flaubert pour la période contemporaine. Il évoque alors les grands moments de l'histoire littéraire, classique pour le dramaturge, romantique et naturaliste pour le romancier.

Cependant, le Conseil général n'a pas encore jugé bon de participer à la souscription, et la somme pour le rachat ne sera pas atteinte. Dans ces conditions, le classement du Pavillon au titre de monument historique, le 11 juin $1906^{22}$, sur décision du ministre de l'Instruction publique, Aristide Briand, précipite les choses : l'inauguration du Pavillonmusée a lieu le 17 juin 1906. L'acte officiel de donation du monument à la municipalité de Rouen est émis le 25 octobre $1907^{23}$.

De quoi la municipalité de Rouen devient-elle propriétaire ? Le rachat et la donation du Pavillon ont lieu au terme d'une période de travaux réalisés par le comité de rachat. Sur l'autel de la reconstitution archéologique et historique, le Comité Flaubert sacrifie la vérité au profit d'une muséographie mythifiante qui consacre l'allée de tilleuls et le pavillon comme lieux d'écriture bucoliques made in Normandy. Aussi l'aménagement du musée, média à part entière porteur d'un discours propre, accompagne-t-il l'histoire littéraire : le musée constitue une illustration tangible qui relaie, voire qui entérine les commentaires des manuels et de la critique. Il façonne un décor de théâtre digne des lithographies romantiques et des descriptions des demeures d'un Chateaubriand ou d'un Rousseau. Par ailleurs, on y installe un musée de reliques et de souvenirs ayant trait à l'écrivain (objets personnels, portraits de famille...) En outre, le Pavillon de 
Croisset offre un aspect que l'on présente au public comme " authentique »: il serait à l'image de ce qu'aurait connu l'écrivain. Le Pavillon fait ainsi partie des monumentsvitrines mis à l'honneur à l'occasion du Millénaire normand, en 1911. Les fêtes sont l'occasion de fédérer la population autour de Corneille mais également autour d'autres gloires locales. Plusieurs « soirée[s] Maupassant » ont lieu au Théâtre-Français au cours de la semaine, tandis que le Théâtre-des-Arts procède au couronnement des bustes des grands auteurs le dimanche 11 juin: l'institution honore les effigies de "Corneille, Bouilhet, Flaubert, etc. ${ }^{24}$ ». Quelques jours plus tôt, le 8 juin, le cortège des invités officiels avait réalisé un parcours qui le conduisait de Saint-Georges de Boscherville (où se trouve une abbaye), au Pavillon de Croisset, en passant par Canteleu ${ }^{25}$.

Mais c'est bien le centenaire de 1921 qui s'annonce comme un événement déterminant pour la petite patrie rouennaise, laquelle entend bien ne pas se laisser damer le pion par Paris. Le comité parisien du centenaire place l'autorité préfectorale de la Seine à la tête des membres du patronage. Ceux-ci représentent les instances nationales littéraires et artistiques (Académie française, Comédie-Française ${ }^{26} . .$. ). C'est alors que la Société des gens de lettres projette l'inauguration du buste de Gustave Flaubert par Auguste Clésinger dans le jardin du Luxembourg, sorte de Panthéon à ciel ouvert où sont réunies les statues des grands personnages de l'Histoire de France. Le jour venu, le 12 décembre 1921, le salon carré du musée du Luxembourg rassemble les autorités officielles des plus hautes institutions concernées : la Présidence de la République avec Jacques Bompard, directeur de cabinet d'Alexandre Millerand, Léon Bérard, ministre de l'Instruction publique et des beaux-arts, ou encore Pau Léon, directeur des Beaux-Arts de la Ville de Paris ${ }^{27}$.

Aussi, à Rouen, Jean Revel et ses soutiens prennent conscience de l'urgence qu'il y a à reprendre les choses en main afin d'assurer à l'enfant du pays « une place définitive [...] parmi nos classiques, à côté des plus hautes gloires des lettres françaises ${ }^{28} »$. Il y a aussi urgence à donner au Pavillon un statut de musée à part entière face à l'exposition parisienne de souvenirs qui a eu lieu en décembre 1921 à la Fédération des artistes ${ }^{29}$. Redoutant la portée limitée que le centenaire pourrait rencontrer en Normandie s'il n'était consacré qu'à Flaubert, le comité célèbre Flaubert aux côtés de son grand ami, Louis Bouilhet, né en 1822. À Rouen, la commémoration donne lieu à des conférences au Théâtre-des-Arts et, à la Bibliothèque municipale, à une exposition de souvenirs ${ }^{30}$. La commémoration se fait également itinérante pour mettre à l'honneur les trésors et les lieux de mémoire attachés au souvenir de Flaubert et à la gloire de la petite patrie. Les tombes des deux amis sont fleuries en présence de la municipalité et du Comité Flaubert. On visite aussi la maison natale de Bouilhet à Cany et le Pavillon de Croisset en présence de Léon Bérard les 21 et 22 mai.

17 Pourtant, l'Académie française s'abstient de participer aux cérémonies : selon les mots de son secrétaire perpétuel, Louis Claude Frédéric Masson, Flaubert n'est rien moins qu'un homme à la mentalité de "vieux médecin de Rouen », adoptant "l'attitude d'un débardeur et d'un chicard ${ }^{31}$ ». Bien vaine alors, la commande, en mai 1922, d'une table en marbre et d'une plaque commémorative à destination de la façade, où figurent la date de la visite de mai 1921 et les noms du ministre, des représentants de la Société des gens de lettres et des élus rouennais. Commémorer une commémoration, tel est le paradoxe auquel on parvient en 1921 sous le propre toit de Flaubert... Les difficultés rencontrées par le conservateur du Pavillon à obtenir l'accord des intéressés à Paris et 
le dédain que ces derniers témoignent dans leur correspondance à l'égard des Amis de Flaubert à Croisset confirment la vanité de l'initiative.

18 En Normandie, la presse locale et le conservateur du Pavillon, Georges A. Le Roy, ne ménagent cependant pas leur peine pour faire connaître le musée. Le Roy, en particulier, remplit ses fonctions avec zèle, aussi bien à travers une correspondance touffue que dans la presse locale et nationale qu'il alimente de dossiers illustrés de plusieurs pages, destinés à attirer le pèlerin et le touriste.

Fig. 1

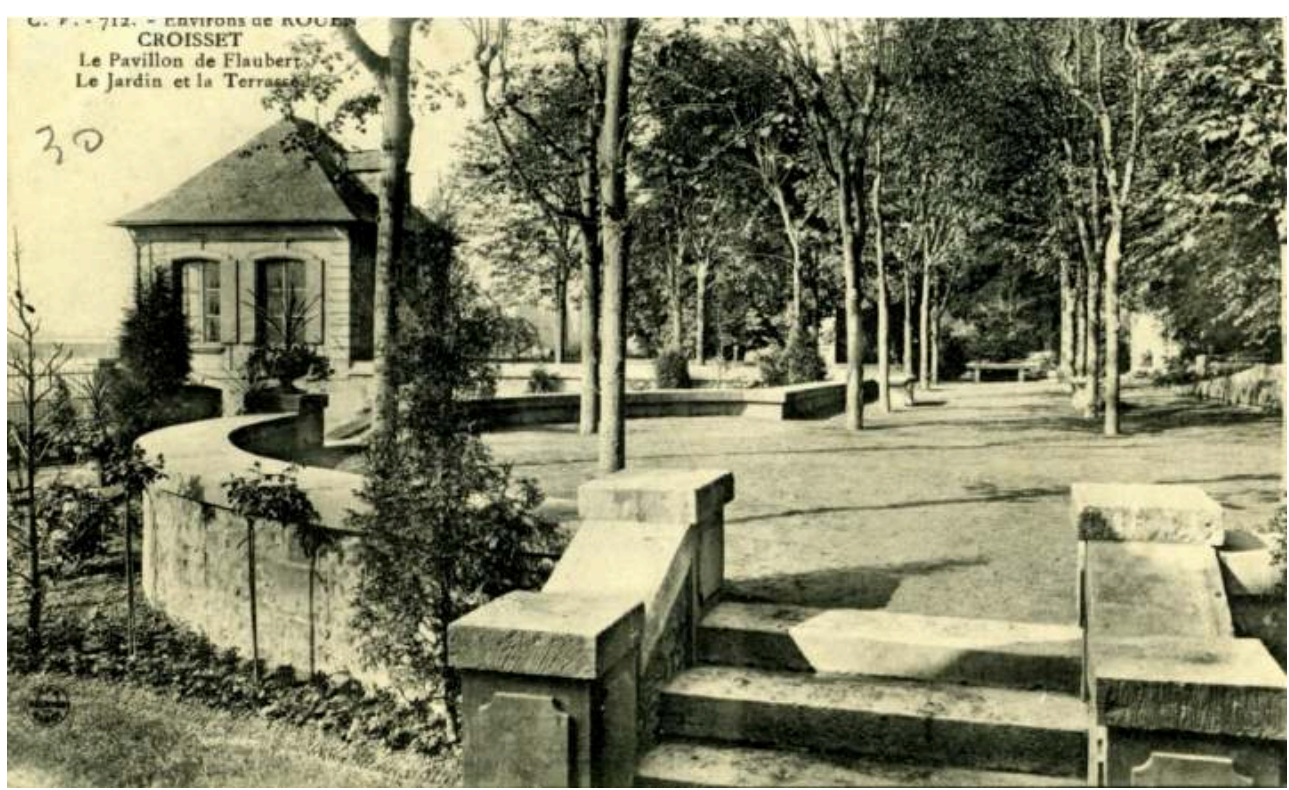

Carte postale du Pavillon de Croisset par Marcel Raitre (env. 1920). Don de A. Poussier. Flaubert E3. Bibliothèque municipale de Rouen.

Fig. 2

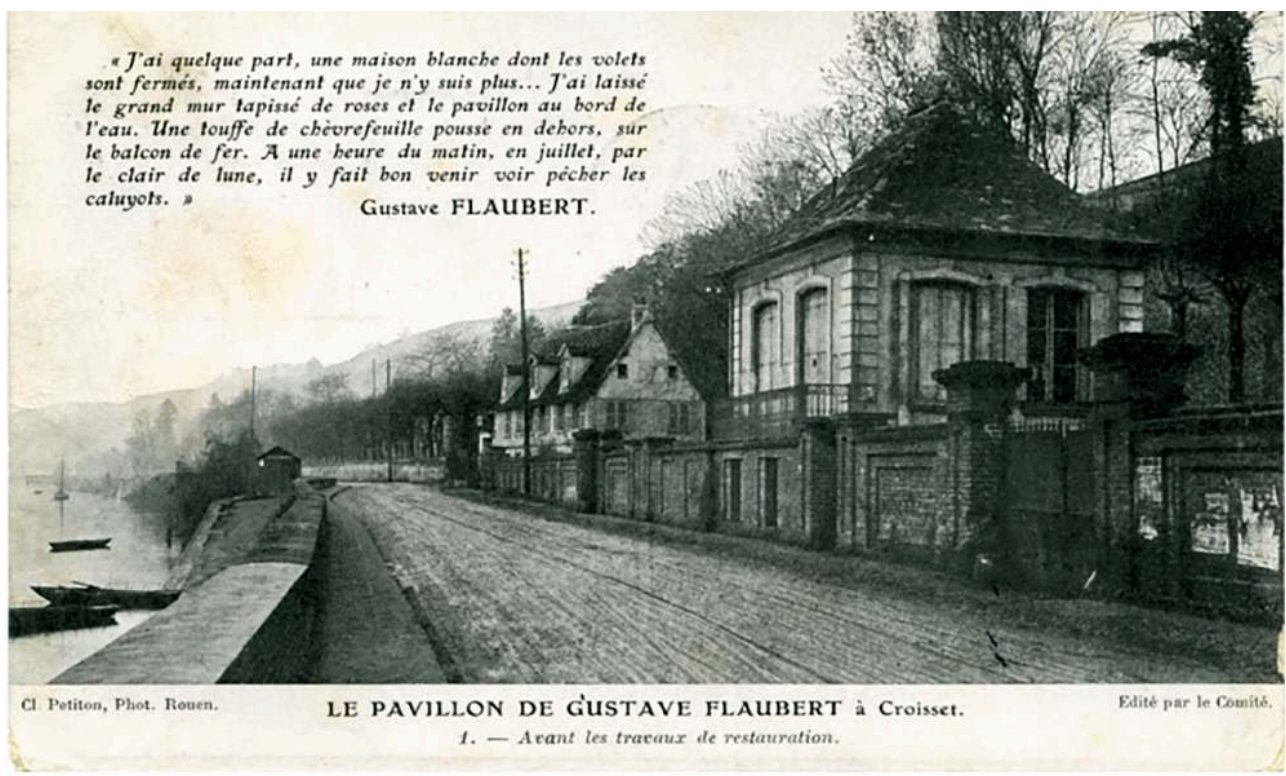

Carte postale du Pavillon de Croisset par Marcel Raitre (env. 1920). Don de A. Poussier. Flaubert E3. Bibliothèque municipale de Rouen. 

patrie normande et du sentiment de fierté nationale attaché au grand homme? La commémoration engendre un grand nombre de textes polémiques et ironiques qui remettent en cause la portée solennelle de l'événement. Un article satirique, intitulé "Chez nous» et paru dans la revue Par chez nous en 1921, écorche lui aussi la commémoration. L'article met en scène un personnage de bibliothécaire au nom désuet et risible, M. Pinchon, qui, en quittant la salle de lecture, « rencontr[e] Flaubert ${ }^{32}$ ». Le portrait du romancier suggère son apparition magique au brave bibliothécaire sous la forme d'une image stéréotypée de l'ermite de Croisset que le professeur du lycée du Havre, Jules Lemaître, présente en classe aux écoliers. L'illusion permet alors de mettre en place le dispositif satirique d'un article aux allures d'apologue. Le fantôme de Flaubert - que Pinchon croit voir s'animer et entendre dire "Qu'on me foute la paix ${ }^{33}$ !»-, sert de prétexte. Il interroge le bien-fondé des manifestations d'admiration que « les “"officiels", un député [...], tout le Conseil municipal », témoignent à l'égard de celui « qu'on avait décrié, détesté, méconnu autrefois ${ }^{34}$ ». Le falot Pinchon devient alors un porte-parole bien commode pour remettre en cause les festivités qui traduisent davantage une instrumentalisation de la mémoire de l'écrivain qu'un hommage véritable, comme on peut le lire dans cet extrait :

Lui, qu'on avait traîné devant la justice de son pays, l'inculpant d'immoralité, peutêtre allait-on déclarer qu'il était le plus pur, le plus classique des écrivains français, peut-être allait-on le proclamer chef d'école, et des thuriféraires importuns essaieraient-ils de se faire un nom en accaparant sa mémoire [...] ?

Devenait-il prophète en son pays, et les gens d'ici, qu'il avait connus, qu'il avait haïs, avaient-ils donc changé ${ }^{35}$ ?

La digression aborde le fond du problème : la littérature et l'écrivain sont devenus, au tournant des $\mathrm{XIX}^{\mathrm{e}}$ et $\mathrm{xx}^{\mathrm{e}}$ siècles, les faire-valoir d'une bourgeoisie marchande mécène, avide de mettre à l'honneur l'identité normande de la petite patrie. Le récit se clôt sur la révélation de l'illusion sur laquelle repose le récit: l'apparition était en réalité liée non pas au surgissement merveilleux de Flaubert, mais à la «rencontre » de la fresque du peintre académique Paul Baudouin, installée dans l'escalier d'honneur de la Bibliothèque municipale de Rouen, en 1889. Énième manifestation commémorative visant à « accaparer » la mémoire de l'écrivain, la fresque est le fruit d'une campagne entamée par Guy de Maupassant, le « disciple » qu'aurait bien connu Pinchon ${ }^{36}$.

L'article souligne par conséquent le caractère très circonstanciel des commémorations à l'occasion d'une date-clef, après laquelle l'enthousiasme collectif - celui des élus du moins - s'épuise rapidement. Ainsi, bien que le monument attire un certain nombre de visiteurs après son inauguration, et en dépit de la bonne publicité dans la presse et en librairie que Dubosc et ses confrères assurent au monument pendant les années 1910, le Pavillon se dégrade, faute d'être entretenu par la mairie et, surtout, à la suite d'un éboulement de falaise pendant la guerre ${ }^{37}$. C'est l'une des raisons pour lesquelles une "Association des Amis de Flaubert » voit le jour le 14 janvier 1914, avec des érudits de Rouen, Raymond Poincaré et des hommes de lettres de renom, tels Maurice Barrès et Henri de Régnier par exemple.

En avril 1921, l'approche du centenaire de la naissance de Flaubert place donc les élus dans une situation pour le moins urgente. Derrière le besoin de doter le musée d'un nouveau gardien après le décès du précédent et d'effectuer des travaux dans son logement de fonction, il est un enjeu autrement plus important: se montrer à la hauteur du grand écrivain qu'est devenu Flaubert dans la région, en France et dans le 
monde entier, en accueillant les visiteurs dans un musée restauré. Bien que le vote d'un crédit (6 000 francs) permette de réaliser les travaux indispensables, de nouvelles réfections s'imposent au cours de la décennie 1920. Pourtant, c'est ailleurs que se joue l'ultime acte de la consécration de Flaubert en Normandie.

Le 22 juin 1923, une chambre-musée de Flaubert est inaugurée à l'Hôtel-Dieu en présence des représentants du préfet et des institutions publiques de la ville ${ }^{38}$. Sur l'initiative du docteur Brunon, fondateur du Musée de la médecine à l'Hôtel-Dieu (devenu propriété municipale en 1921), de M. Poussier, pharmacien-chef de l'HôtelDieu et de Louis Bertrand, académicien, la reconstitution du sanctuaire offre aux pèlerins la visite de la pièce et du cabinet de toilette adjacent (celui de Mme Flaubert) à partir de quelques documents d'archives. Les meubles - certes d'époque ${ }^{39}$ - n'ont pas appartenu aux Flaubert. Toutefois, pour ajouter un cachet à la pièce, des portraits du père, des frères et de Gustave à différents âges et par des artistes de renom, ornent les murs. Mais il ne s'agit pas d'originaux ${ }^{40}$. La reconstitution procède en ce sens de la localisation et de la réification d'un imaginaire auquel l'écrivain est renvoyé dans la critique littéraire: l'univers médical auquel le style de l'écrivain, au scalpel, emprunterait dans une large mesure ${ }^{41}$.

Fig. 3

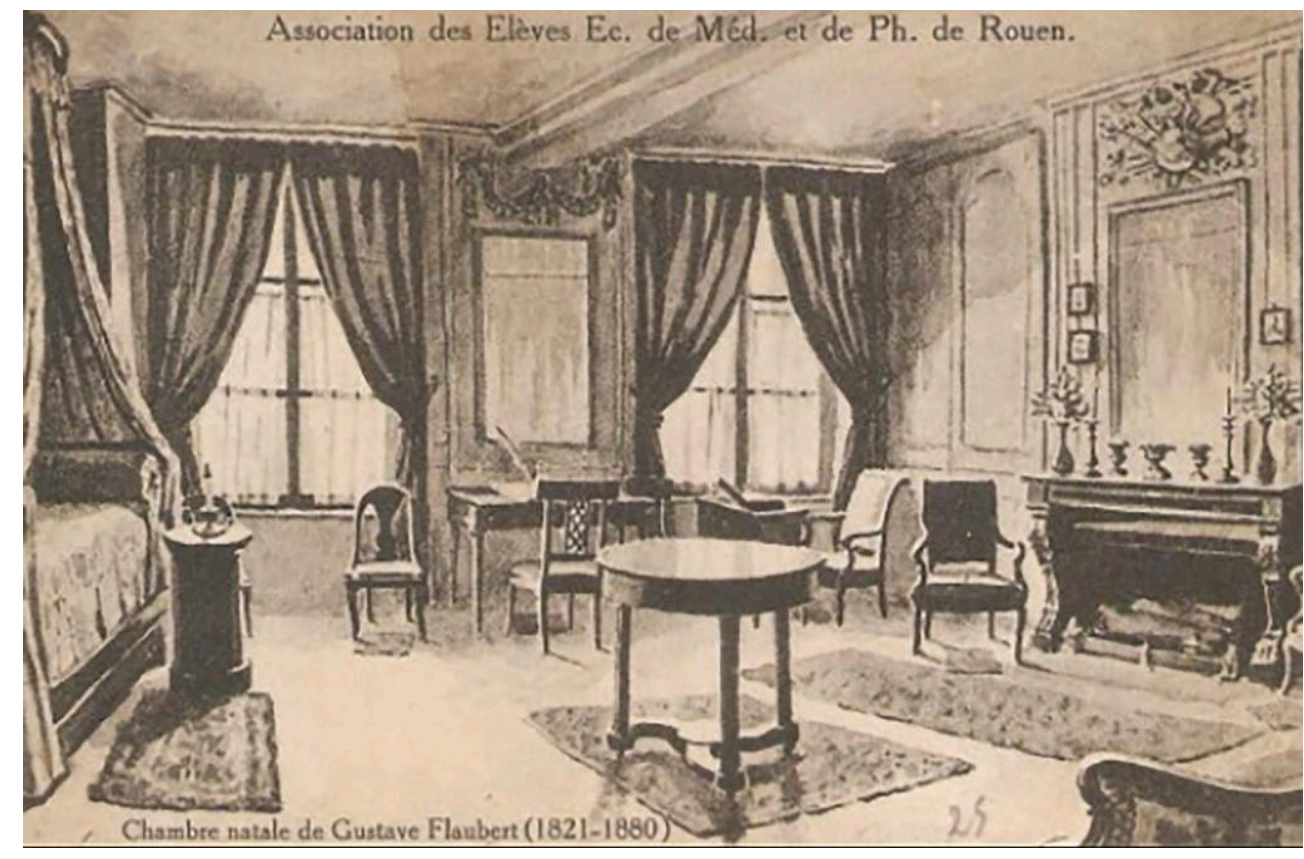

Carte postale datant des années 1920-1930 figurant la reconstitution de la chambre natale de Flaubert à Rouen ${ }^{42}$.

Bibliothèque municipale de Rouen (Inv. PF 455 - Flaubert E3 m 18).

Toutefois, Louis Dubreuil annonce d'emblée son refus de se soumettre au rituel du discours apologétique pour s'interroger sur le statut du grand homme que l'inauguration d'un nouveau mémorial renforce un peu plus : « Je tiens à déclarer que je ne ferai pas de discours et je commencerai en présentant les excuses de celui qui n'a pas pu venir parmi nous : G. Flaubert ${ }^{43} »$. Entamant une prosopopée qui ne s'enclenche jamais véritablement, le maire s'interroge :

Je crois que s'il était présent, G. Flaubert s'excuserait d'avoir légué le culte de sa mémoire à cette ville qui, ayant donné naissance au plus grand des poètes 
tragiques, et à l'un des plus grands peintres du siècle dernier, possède le privilège d'avoir donné au pays un de ses plus grands romanciers.

Flaubert n'aimait pas Rouen, ou feignait de ne pas l'aimer, cependant il est resté toute sa vie fidèle à la Normandie qui l'avait vu naître et nous pourrions le définir comme l'a défini Taine "une espèce de moine qui habite près de Rouen et qui a composé une histoire édifiante : Emma ou les dangers de l'inconduite ».

Quoi qu'on puisse dire, il faut reconnaitre que le chef-d'œuvre de Flaubert a pour cadre Rouen et ses environs. S'il s'en fut vers les rives méditerranéennes, c'est qu'il ressentait comme tous les Barbares du Nord l'attrait des splendeurs bleutées de la mer Méditerranée ${ }^{44}$.

L'élu ne clôt pas son discours sur cette note poétique forcée et ironique. Soucieux de concilier, voire de réconcilier, la mémoire du romancier avec sa ville et ses contemporains, le maire excuse les deux partis :

Le mépris qu'il croyait que Rouen lui inspirait n'était qu'une des formes de l'éternelle histoire du génie qui se sent mal à l'aise dans la réalité et qui ne sait pas se laisser aller avec philosophie sur l'Océan sans borne de la bêtise humaine. Il y a toujours chez les contemporains une impossibilité de comprendre le génie qui éclôt au milieu d'eux ${ }^{45}$.

En effet, la commémoration doit maintenir un esprit de concorde autour du souvenir du grand écrivain. Toutefois, le Rouen qui rit, journal d'étudiants, constate au sujet des «Fêtes du Centenaire de Flaubert »:

Il semble dans les circonstances critiques que nous traversons, que la France éprouve le besoin, pour faire face aux difficultés de l'heure, de s'appuyer sur ses fils avec plus d'abandon. C'est surtout auprès de ceux qui sont morts qu'elle trouve le plus utile réconfort. C'est pourquoi notre ville vient de célébrer avec une pompe toute particulière le centenaire du grand écrivain et du grand Français que fut Gustave Flaubert ${ }^{46}$.

Il faut lire dans ses lignes la critique que les étudiants du journal adressent à leurs pères et aux élus, eux qui privilégient le culte de la mémoire des écrivains morts (et des anciens combattants) au détriment d'une jeunesse qui aspire à tout autre chose qu'à inaugurer les chrysanthèmes... En effet, une question se pose : les commémorations en faveur de Flaubert n'interviennent-elles pas à une époque où le "sacre de l'écrivain » a déjà vécu ses plus belles heures? Le culte de la personnalité de l'écrivain, réifié dans le musée, est-il encore entretenu et partagé par la communauté normande et nationale ? Ne tient-il pas davantage à un folklore, celui du " grand écrivain ", de l'ermite retiré en sa "chartreuse littéraire " dans un paysage bucolique? N'est-il donc pas devenu davantage une curiosité et une matière à écrire un bon mot qu'un lieu de pèlerinage solennel ? Dans ce contexte, pour qui le Pavillon du grand écrivain et de l'auteur a-t-il encore quelque valeur: pour quelques érudits (non rebutés par la muséographie truquée), pour les admirateurs composant le Comité Flaubert, pour les notables rouennais et les hommes politiques ? N'est-il pas l'« étalage de la vanité humaine ", selon les termes que Jean Jaurès emploie lors de sa visite en voyant les autographes des « riches et prétentieux » académiciens ${ }^{47}$ ?

De ce point de vue, l'exemple du Pavillon de Flaubert met en lumière les limites du processus d'appropriation mémorielle qui se rapporte à l'ermite de Croisset. Ainsi, bien que le comité de rachat du Pavillon ouvre le groupe au-delà des proches et des amis de l'écrivain en 1905-1907 et ce grâce à une souscription publique, Toutain-Revel sanctuarise le périmètre des soutiens envisagés. Écrivains, artistes, autorités politiques, institutions des Belles-Lettres et des Arts parisiennes et rouennaises, bourgeoisie normande : tels sont les «amis» de Flaubert que dénombre le notaire. Pourtant, les 
décennies 1900-1920 marquent l'entrée du romancier dans les manuels scolaires, dans les guides de voyage, ainsi que sa reconnaissance par une large frange de la critique littéraire. Mais, à la différence de Corneille, père spirituel de tous les Français, l'auteur de Salammbô demeure un écrivain d'élite pour les élites.

Ainsi, le développement des sociétés d'amis, stimulé par les projets de rachat des maisons de Flaubert, invite à reconsidérer le rôle des sociétés savantes et des hommes de lettres, écrivains et artistes dans la patrimonialisation de l'écrivain et tout particulièrement dans l'organisation de ses commémorations dans les années 1910-1920. En effet, ils sont relayés par les groupes d'«amis». Ces groupes comme les sociétés d'amis continuent de représenter les défenseurs les plus fidèles de la mémoire des écrivains et de leurs monuments en attendant la mise en œuvre d'une politique publique volontariste de célébration de la mémoire des gloires littéraires et des fonds patrimoniaux qui leur sont attachés.

\section{NOTES}

1. J. Leerssen et A. Rigney (dir.), Commemorating Writers in Nineteenth-Century Europe: NationBuilding and Centenary Fever, Basingstoke, Palgrave Macmillan, 2014.

2. P. Boudrot, "Voltaire 1878. Commemoration and the Creation of Dissent », ibid., p. 152.

3. Voir par exemple Hélène Combis, "Peut-on, doit-on commémorer Maurras (et Céline) ?", France culture, 2 février 2018. En ligne: <https://www.franceculture.fr/litterature/celine-etmaurras-peuvent-ils-etre-commemores> (consulté le 13 février 2018).

4. Voir notamment G. Flaubert, Lettre de M. Gustave Flaubert à la municipalité de Rouen au sujet d'un vote concernant Louis Bouilhet, Paris, Michel Lévy, 1872.

5. Yvan Leclerc a consacré un article au sujet; il propose une chronologie remarquable des événements du vivant de Flaubert à nos jours : Y. Leclerc, "Flaubert "définitivement réconcilié" avec Rouen? », Études normandes, M. Dufour-Maître et Y. Leclerc (dir.), « Normands de plume », p. 41-50.

6. C. Franklin-Grout, Heures d'autrefois. Mémoires inédits. Souvenirs intimes et autres textes, textes établis, présentés et annotés par Matthieu Desportes, Rouen, Presse universitaires de Rouen et du Havre, 1999, p. 139, cité par J. Robert, «Les "normandismes indéfinis" de Gustave Flaubert ", Études normandes, ouvr. cité, p. 31-40:p. 31.

7. C. Franklin-Grout, Heures d'autrefois. Mémoires inédits. Souvenirs intimes et autres textes, textes établis, présentés et annotés par Matthieu Desportes, ouvr. cité, p. 31-40 : p. 31.

8. J. Robert, "Les "normandismes indéfinis" de Gustave Flaubert », art. cité, p. 37. M. du Camp, Souvenirs littéraires, Paris, Hachette, [1882] 1906, p. 180.

9. A. Thibaudet, Gustave Flaubert, Paris, Gallimard, 1935, p. 9. Paraît en 1922 une première version : Gustave Flaubert. 1821-1880. Sa Vie - Ses Romans - Son Style.

10. Ibid., p. 10.

11. "Aux sources de la thèse de Ry ", Les Amis de Flaubert, bulletin $n^{\circ} 25,1964$, p. 40. En ligne : <http://www.amis-flaubert-maupassant.fr/article-bulletins/025_040/> (consulté le 20 mai 2017). Voir aussi Jean-Marie Privat, "Emma à Ry. Notes de recherche », ethnographiques.org, $\mathrm{n}^{\circ} 5$, avril 2004, en ligne : <https://www.ethnographiques.org/2004/Privat> (consulté le 15 janvier 2020) et 
Jean-Marie Privat « ... votre pensée, s'enlaçant à la fiction. Ry/Bovary, une médiation littéraire grand public ", Recherches et Travaux $\mathrm{n}^{\circ}$ 96, 2020.

12. A. Thibaudet, Gustave Flaubert, ouvr. cité, p. 92.

13. D. Michon, « Histoire des Amis de Flaubert de 1880 à 1980 », Bulletin Flaubert-Maupassant, 2012, $\mathrm{n}^{\circ}$ 27, p. 140-164: p. 141. L'auteur réalise une thèse sous la direction de C.-I. Brelot: Une mémoire conflictuelle: Gustave Flaubert en France (1821-2008). En ligne: <http://www.theses.fr/s102813> (consulté le 22 mars 2017).

14. G. Flaubert, Lettre de M. Gustave Flaubert à la municipalité de Rouen..., ouvr. cité, p. 142.

15. «Au niveau du visage de l'allégorie, sur le fond, sont inscrites différentes œuvres de Flaubert : Madame Bovary, Salammbô, La Tentation de Saint Antoine, L'Éducation Sentimentale, Bouvard et Pécuchet, Le Candidat ", notice du portail des collections des musées de France, en ligne: $<$ http://www.culture.gouv.fr/public/mistral/joconde_fr?

ACTION=CHERCHER\&FIELD_1=REF\&VALUE_1=07340001776> (consulté le 31 mai 2016).

16. Edmond de Goncourt, Journal. Mémoires de la vie littéraire, Paris, t. VIII (1889-1891), Paris, G. Charpentier et E. Fasquelle, 1895, p. 185.

17. Voir aussi Y. Leclerc, « Flaubert "définitivement réconcilié” avec Rouen », art. cité, p. 45-46.

18. "En tout, une vingtaine de Parisiens de marque, dans les lettres et le reportage, et une fête avec tente pour les autorités et musique de foire, comme pour les Comices agricoles de Madame Bovary »: Edmond de Goncourt, "Dimanche 23 novembre 1890 », Journal des Goncourt: mémoires de la vie littéraire, $3^{\mathrm{e}}$ série, vol. 2 (1889-1891), Paris, Bibliothèque Charpentier, G. Charpentier et E. Fasquelle, 1895, p. 180.

19. D. Michon, « Histoire des Amis de Flaubert de 1880 à 1980 », art. cité, p. 143.

20. Notice, site officiel de la manifestation « Flaubert dans la ville», Rouen 17 avril-31 août 2015, en ligne: <https://flaubert-danslaville.univ-rouen.fr/4-place-des-carmes> (consulté le 31 mai 2016).

21. P. Toutain, «Le Pavillon de Flaubert », Le Journal de Rouen, 26 novembre 1904, p. B.

22. Arrêté de classement au titre des monuments historiques du Pavillon de Gustave Flaubert, à Croisset, commune de Canteleu (Seine-Inférieure), Flaubert B1-B3. Bibliothèque municipale de la Ville de Rouen.

23. Extrait du registre des délibérations, séance du vendredi 8 novembre 1907. Flaubert B1-B3, Bibliothèque municipale de la Ville de Rouen.

24. Fêtes du Millénaire normand. Congrès ( 5 au 10 juin 1911). Organisation et programme, Rouen, impr. L. Wolf, 1911 [s. p.].

25. Ibid.

26. Centenaire de Gustave Flaubert, Paris, Renouard, 1921, p. 41 et suiv. Flau D3-16. Bibliothèque municipale de Rouen.

27. Ibid., p. 1 et suiv.

28. « Le Pavillon de Flaubert », Le Journal de Rouen, art. cité.

29. "L'exposition Gustave Flaubert ", Le Figaro, 8 décembre 1921, "Pupitre de G. Flaubert", Flaubert B4-19., Bibliothèque municipale de Rouen.

30. Brouillon du programme des festivités adressé à G.-A. Leroy par la municipalité de Rouen, "Centenaire de Flaubert (et Bouilhet), 1921. Cérémonie à Rouen (Comité Flaubert). Ordre chronologique ", Flaubert D3-9, Bibliothèque municipale de Rouen.

31. Lettre de Frédéric Masson reproduite dans Comœdia, juin 1921, extrait cité par D. Michon, art. cité, p. 151.

32. P. Mérat, "Chez nous ", Par chez nous. Revue normande de littérature et d'art, mai 1921, $\mathrm{n}^{\circ} 7$, p. 13. Don de la rédaction pour le Centenaire Flaubert et Bouilhet. [Inv. PF 378], Flaubert D3-1, Bibliothèque municipale de Rouen.

33. Ibid., p. 13.

34. Ibid., p. 13. 
35. Ibid., p. 13.

36. Ibid., p. 13.

37. Lettre du 6 juin 1919 de l'architecte de la Ville au maire au sujet d'un éboulement survenu le 21 avril 1917. «Pavillon Gustave Flaubert - délibérations 1904 à 1967 - travaux 1906 à 1960 musée des Antiquités - délibérations 1832 à 1922 et travaux 1832 à 1937 + Centre d'art contemporain 46W583 ", archives municipales de la Ville de Rouen.

38. E.-H. Guitard, «La chambre natale de Flaubert », Revue d'histoire de la pharmacie, $19^{\mathrm{e}}$ année, $\mathrm{n}^{\circ} 76,1931$, p. 281-282.

39. «À l'Hôtel-Dieu. Inauguration de la chambre où naquit G. Flaubert », La Dépêche, 23 juin 1923, FLAU D2-2, Bibliothèque municipale de Rouen.

40. Ibid.

41. Voir R. Dumesnil, Flaubert, son hérédité, son milieu, sa méthode, Paris, Société française d'imprimerie et de librairie, 1906; Françoise Grauby, Le Corps de l'artiste: discours médical et représentations littéraires de l'artiste au XIX siècle, Lyon, Presses universitaires de Lyon, 2001, p. 34. L'auteur rappelle à propos que Sainte-Beuve façonne l'image de la plume-scalpel dans ses Causeries.

42. Dessin de la «Chambre où naquit Gustave Flaubert» par J. Savalle-L, photographie 208 x $142 \mathrm{~mm}$ dans Bulletin de l'Association des anciens élèves de l'École de Médecine et de Pharmacie de Rouen [1924] [Inv. PF 455]. Flaubert E3 m 18, Bibliothèque municipale de Rouen.

43. «À l'Hôtel-Dieu. Inauguration de la chambre où naquit G. Flaubert », art. cité.

44. Ibid.

45. Ibid.

46. "Les Fêtes du Centenaire de Flaubert. Visite à Rouen du Ministre de l'Instruction Publique ", Le Rouen qui rit, $1^{\mathrm{er}}$ juin 1921, p. 1. Flaubert $\mathrm{D} 3-6$, Bibliothèque municipale de Rouen.

47. $\mathrm{P}$. Mérat, «Jaurès chez Flaubert », Rothomagus, août 1914, $\mathrm{n}^{\circ} 1$. Flaubert $\mathrm{B}$ 2-14, Bibliothèque municipale de Rouen.

\section{RÉSUMÉS}

Flaubert a été un porte-voix des critiques et des opposants aux commémorations. L'écrivain ne s'est pas seulement érigé contre ces grand-messes collectives ; après sa mort, il apparaît aussi comme un auteur non " commémorable " aux autorités de la " petite patrie » normande dont il est pourtant l'un des grands noms avec Pierre Corneille et Guy de Maupassant. Cet article analyse les commémorations qui lui ont été consacrées après sa mort, à Paris et en Normandie, en plaçant l'accent sur l'ancrage géographique de ces festivités et sur leur caractère polémique.

Flaubert can be considered as a spokesperson for the opponents to commemorations. The writer not only stood up against these collective high-masses; after his death, he was also seen as a writer who was not eligible for such honors according to the point of view of local authorities in Normandy despite his great reputation among other local celebrities such as Pierre Corneille and Guy de Maupassant. This paper analyses several commemorations of Flaubert in Paris and in Normandy: it particularly highlights the question of their territorial anchoring and their polemical dimension. 
INDEX

Keywords : Flaubert, cultural heritage, writer's museum, literary memory

Mots-clés : Flaubert, commémoration, maison-musée, patrimoine, mémoire littéraire

\section{AUTEUR}

\section{MARIE-CLÉMENCE RÉGNIER}

Ancienne élève de l'École normale supérieure de Paris, agrégée de lettres modernes et diplômée de Sciences Po Paris, Marie-Clémence Régnier a soutenu une thèse en littérature française sous la direction de Françoise Mélonio et de Florence Naugrette à la Sorbonne sur la genèse des maisonsmusées d'écrivains en France, sous le titre : «Vies encloses, demeures écloses. Le grand écrivain français en sa maison-musée (1879-1937) ». Elle est maître de conférences à l'université d'Artois. Ses recherches portent sur la construction de la figure de l'écrivain et de l'histoire littéraire dans les maisons-musées, les musées et les expositions littéraires, et plus largement sur les interactions entre littérature, patrimoine et culture médiatique moderne depuis le XVIII ${ }^{\mathrm{e}}$ siècle. 